\title{
Silicone toe prosthesis using silicone rings as a mode of retention- A case report
}

\author{
Manisha Singh ${ }^{1,}$, Siddharth Mehta ${ }^{2}$, Dhanya Kumar ${ }^{3}$, Nandeeshwar D. B. ${ }^{4}$ \\ ${ }^{1}$ PG Student, ${ }^{2}$ Senior Lecturer, ${ }^{3}$ Professor, ${ }^{4}$ Professor \& HOD, Dept. of Prosthodontics and Crown \& Bridge, Bapuji Dental \\ College, Davanagere, Karnataka, India
}

*Corresponding Author:

Email: manishasingh13391@gmail.com

\begin{abstract}
Silicone cosmetic restorations allow people with toe amputations to wear a passive prosthesis that looks almost identical to their real toe. These restorations appear so realistic that they are virtually unnoticed by others. The silicone prosthesis are able to restore the appearance of the lost toe and provide some passive function. The challenges faced by maxillofacial prosthetic patients range from a fear of social rejection to the economic burden of losing employment. This case report describes an effective approach of rehabilitation of a partially amputed toe using a custom made silicone prosthesis using ring structure for optimal retention and use of silicone prosthesis can fulfil patients esthetics as well.
\end{abstract}

Keyword: Toe prosthesis, Maxillofacial prosthesis, Ring substructure.

\section{Introduction}

Challenges faced by maxillofacial prosthetic patients ranges in from of psychological, social, and economic condition. ${ }^{1,2}$ These manifestations result in clinical depression. ${ }^{3}$ Several studies noted simple correlation between the volumetric expanse of maxillofacial defects and depressive severity. ${ }^{4,5}$ There is improvement in psychological behaviour of patient after maxillofacial prosthetic rehabilitation.

Silicone prosthesis is ideal method for restoring maxillofacial defects other than implant when it comes to economical and surgical procedure. $^{6}$ Polydimethylsiloxanes have been used extensively for biomedical applications and especially in restoring facial defects with prostheses. It is room temperature vulcanized (RTV) elastomers in which tin compounds are the catalyst. ${ }^{7,8}$

This case report describes a cost effective approach of rehabilitation of a partially amputed toe using a custom made silicone with ring structure for optimal retention and esthetics.

\section{Clinical Report}

A 42 year male patient presented with chief complain of missing toe due to trauma followed by amputation 15 years back. The patient was very eager to get the amputed toe rehabilitated due to social life being affected.

On inspection the defect was present in right index toe. The defect was till mid-level of index toe leaving behind scar tissue formation. There were no signs of discolouration, pulsating veins, and oedema noticed (Fig. 1). On palpation of defect the distal area was bulbous which was compressible in nature and no bony undercuts were present.

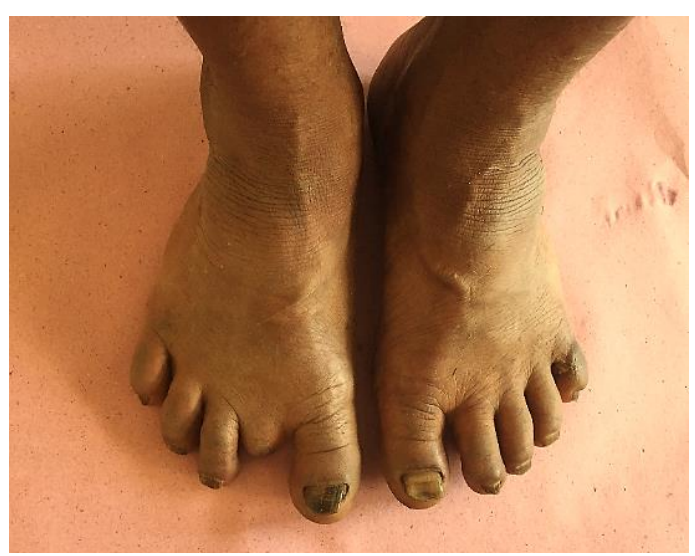

Fig. 1: Showing defect of right index toe

Treatment option presented to patient includes implant supported maxillofacial prosthesis and custom made silicone prosthesis. As patient was not ready to undergo any type of surgical intervention, the final treatment option of custom made silicone prosthesis was selected and to get optimal retention silicone ring structure were used in adjacent toes.

The impression of the index toe was made with irreversible hydrocolloid alginate (3M ESPE, Bangalore, India-560100) and the model was poured with type 3 gypsum product (Kalabhai Kalstone). An impression of the index toe of the left foot was made with polyvinyl siloxane putty impression material $(3 \mathrm{M}$ ESPE, soft putty, Bangalore, India-560100) which served as a donor toe (Fig. 2). The impression was poured with modelling wax (Elite dental products, Nanded, India - 431605) and retrieved on cooling. (Fig. $3)$. 


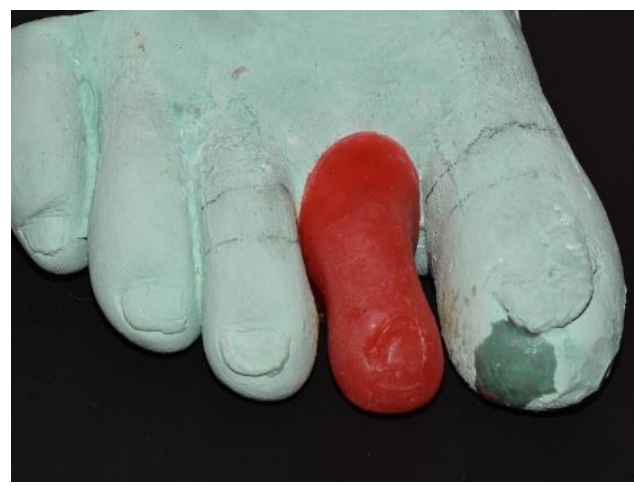

Fig. 2: Wax pattern try in on stone model

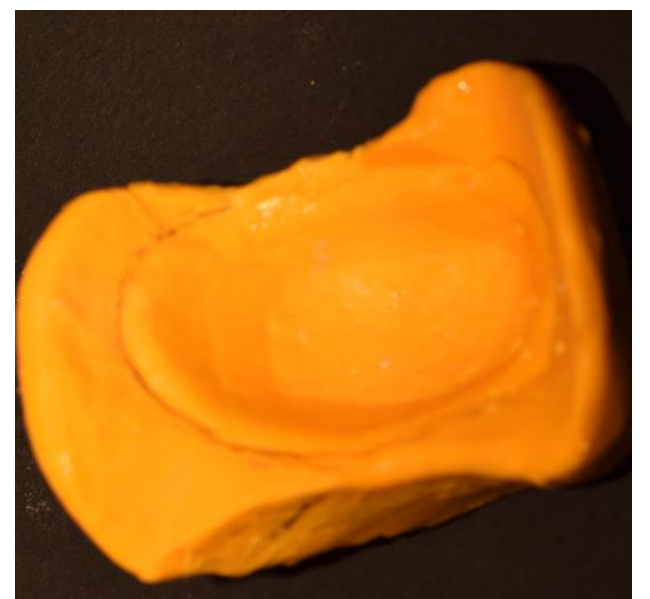

Fig. 3: Left index toe impression made using soft putty

Scoring was done in stone model on adjacent toes to obtain ring which will aid in retention. Measurements from stone model of adjacent toes and missing toe was measured using thread and same measurements were transferred to make soft putty model of whole prosthesis . Wax up was done on soft putty model (Fig. 4) and the whole unit was flasked using two pour technique. Dewaxing was done and the mould was inspected for any impurities of broken fragments of dental stone (Fig. 5). A layer of soap solution was applied all through the surface of the mould which acted as a separating media. Cosmesil RTV Maxillofacial silicone (Cosmesil series material, Principality Medical Ltd, South Wales, UK) was used for the fabrication of prosthesis. The silicone is available in Part A and Part B (Part A: Part B to be mixed in the ratio of 10:1). Intrinsic skin colour matching was done chair side in presence of the patient. Intrinsic skin shade matching was done (Fig. 6). After satisfactory shade matching was obtained, Part B was mixed and the silicone was left overnight for curing at room temperature as per the manufacturer's recommendations.

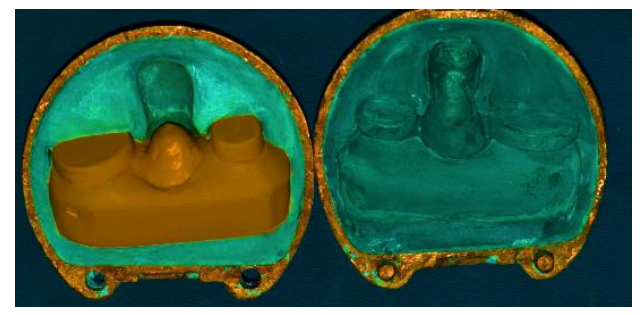

Fig. 4: Flasking and dewaxing

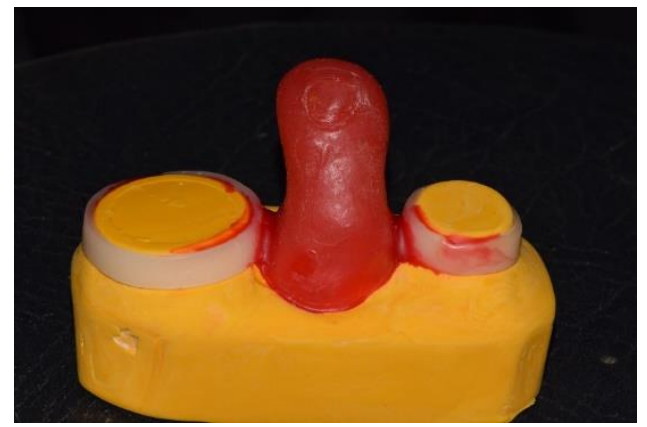

Fig. 5: Wax up of putty model with missing index toe

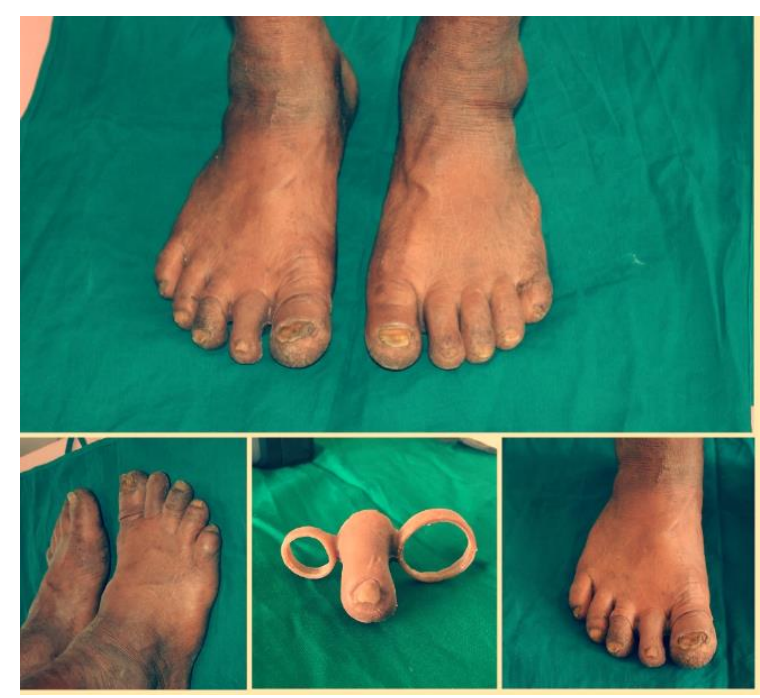

Fig. 6: Final prosthesis

The polymerised toe prosthesis was retrieved from the flask and was inspected for any surface irregularities, nodules, fins and dental stone remnants. The prosthesis was finished with silicone finishing burs. The extrinsic staining was done chair side in presence of the patient. The prosthesis was inserted and checked for the color matching, orientation of the prosthesis and its camouflage with the remaining residual stump .Post insertion instructions were given regarding the maintenance of the prosthesis.

\section{Summary}

Maxillofacial prosthetic restoration brings about responses of the whole person. These can be measured indirectly through the changes evoked in the self-image and in the strength of positive optimism. Ultimately, the 
skill and competence of clinicians along with the fortitude of patients defines the success of maxillofacial prostheses. The advent of room temperature vulcanizing silicon (RTV) has proved to be a boon in restoring esthetics.

\section{Conclusion}

It is the god given right of every human to appear human. Rehabilitating the defects of the toe with an artificial prosthesis can be rewarding and satisfying for a maxillofacial prosthodontist. Despite of the availability of the advanced skills, best of materials and laboratory support, sometimes the anatomy of the defect may be a hindrance in furnishing a better prosthesis. The toe prosthesis with custom made ring substructure was functionally adequate and esthetically acceptable by the patient.

Funding: No funding sources.

Conflict of interest: None declared.

\section{References}

1. Bailey LW, Edwards D. Psychological considerations in maxillofacial prosthetics. J Prosthet Dent 1975;34:533-8.
2. Turns D, Sands RG. Psychological problems of patients with head and neck cancers. J Prosthet Dent 1978;39:6873.

3. Rozen RD, Ordway DE, Curtis TA, Cantor R. Psychosocial aspects of maxillofacial rehabilitation. I. The effect of primary cancer treatment. J Prosthet Dent 1972;28:423-8.

4. Sykes BE, Curtis TA, Cantor R. Psychosocial aspects of maxillofacial rehabilitation. II. A long-range evaluation. $J$ Prosthet Dent 1972;28:540-5.

5. Roefs AJ, van Oort RP, Schaub RM. Factors related to the acceptance of facial prostheses. J Prosthet Dent 1984;52:849-52.

6. Habal, M. 3.; The biological basis for the clinical application of the silicones. Arch Surg 1984;119:843-8.

7. Craig, R. G., Koran, A. \& Yu, R., Elastomers for maxillofaciai applications. Biomater 1980;1:112-17.

8. Polyzoisa G, Stafford G.D, Winter R, A study of some mechanical properties of an RTV polydimethylsiloxane for extraoral maxillofacial prostheses. Clin Materials 1992;9:21-9.

How to cite the article: Singh M., Mehta S., Kumar D., B. Nandeeshwar. Silicone toe prosthesis using silicone rings as a mode of retention- A case report. Ann Prosthodont Restor Dent 2018;4(3):87-89. 